\title{
Towards Road Safety
}

\section{A Social Perception}

\author{
Bruno Fernandes $^{1(\bowtie)}$, José Neves ${ }^{1}$, Henrique Vicente ${ }^{2}$, \\ and Cesar Analide ${ }^{1}$ \\ ${ }^{1}$ Department of Informatics, Centro ALGORITMI, \\ University of Minho, Braga, Portugal \\ bruno.fmf.8@gmail.com, \{jneves, analide\}@di.uminho.pt \\ 2 Department of Chemistry, University of Évora, Évora, Portugal \\ hvicente@uevora.pt
}

\begin{abstract}
This meta-analytic study analyzes psychological research focusing on road safety as proposed by the Vulnerable Road Users (VRUs) approach, paying attention to attributes such as VRUs habits, environment and things. Aiming to consider VRUs as active and proactive actors, with differentiated feelings and behaviours, we were required to integrate in our study the social factors that characterize each VRU. In fact, many studies have shown how social factors may put at risk a human being, with special incidence in socioeconomic groups where lower social positions correlate with an increased exposure to danger. Therefore, diminishing social inequities by addressing the social factors that motivate them developed into an explicit goal to enhance road safety. Undeniably, one way to examine how such factors affect injury is to study them at different degrees. Hence, focusing on such attributes, which may be indicative of inequalities in social status, we are able to quantify their degree of disorder in terms of an entropic metric. The computational framework is to be understood under a mathematical logic approach to computing.
\end{abstract}

Keywords: Internet of people $\cdot$ Entropy

Knowledge representation and reasoning $\cdot$ Social machine

Vulnerable road users $\cdot$ Road safety 